\title{
Blade Exit Angle Effects on Performance of a Standard Industrial Centrifugal Oil Pump
}

\author{
W. G. $\mathrm{Li}^{\dagger}$ \\ Department of Fluid Machinery, Lanzhou University of Technology \\ 278 Langongping Road, 730050 Lanzhou, P R China \\ †Corresponding Author Email: Liwg38@yahoo.com.cn
}

(Received November 8, 2009; accepted January 12, 2010)

\begin{abstract}
The effects of blade discharge angle on the performance of a standard industrial centrifugal oil pump of type 65Y60 were investigated experimentally as the pump handled both water and viscous oil. A one-dimensional hydraulic loss model was established to identify such effects mathematically. The effects have been estimated analytically by using the model at various viscosities. The results showed that the blade discharge angle has significant but equal influence on the head, shaft power and efficiency of the centrifugal oil pump at various viscosity conditions. For any viscosity, the total hydraulic loss in the impeller and volute rises with increasing blade exit angle. The diffusion loss in and behind the impellers as well as the friction loss in the volute are noticed in the pump, especially for highly viscous liquids. The hydraulic loss in the impellers is about 0.8-0.6 times the loss in the volute. In order to improve the pump performance, the hydraulic loss in the volute must be kept as small as possible.
\end{abstract}

Keywords: Centrifugal pump, Centrifugal oil pump, Performance, Blade angle, Hydraulic loss, Disk friction loss

\section{NOMENCLATURE}

$b_{1} \quad$ width of blade inlet

$b_{2} \quad$ width of blade outlet

$b_{3} \quad$ width of volute

$C_{M} \quad$ torque coefficient due to disc friction

$D_{1} \quad$ diameter of blade inlet

$D_{2} \quad$ diameter of blade outlet

$D_{3} \quad$ diameter of circle tangential to volute tongue tip

$D_{8} \quad$ equivalent diameter of volute throat

$D_{89} \quad$ mean diameter of $D_{8}$ and $D_{9}$

$D_{9} \quad$ diameter of discharge nozzle

$D_{h} \quad$ hydraulic diameter

$D_{w} \quad$ diameter of wear-ring

$d H / d Q \quad$ slope of head-flow rate curve

$F_{0} \quad$ cross-sectional area of section 0-0

$F_{2} \quad$ exit area of impeller

$F_{8} \quad$ throat area of volute

$F_{9} \quad$ cross-sectional area of nozzle exit

$F_{m} \quad$ mean area of $F_{0}$ and $F_{8}$
$Q_{t} \quad$ theoretical flow rate through impeller

$Q_{W} \quad$ flow rate of water

Re Reynolds number

$R_{2} \quad$ impeller disc Reynolds number

$R e_{d} \quad$ Reynolds number in nozzle

$R_{w} \quad$ radius of wear-rings on impeller

$S_{u 2} \quad$ tangential blade thickness at outlet

$t$ distance between casing and impeller shroud or hub

$T$ temperature of fluid

$u_{2} \quad$ impeller tip speed

$V_{3} \quad$ mean velocity in volute

$V_{89} \quad$ mean velocity through area of $\pi D_{89}^{2} / 4$

$V_{9} \quad$ mean velocity through nozzle exit

$V_{m 2} \quad$ meridian velocity at outlet

$V_{u 2} \quad$ tangential absolute velocity at outlet

$W \quad$ mean relative velocity of $W_{1}$ and $W_{2}$

$W_{1} \quad$ relative velocity at entrance of impeller

$W_{2} \quad$ relative velocity at exit of impeller

$W_{2 \infty}$ relative velocity at the exit of impeller with 
$f_{\text {geo }} \quad$ impeller shape factor for disk friction loss

$g$ acceleration due to gravity

$h_{l} \quad$ total hydraulic loss

$h_{i d} \quad$ expansion loss in impeller

$h_{i e} \quad$ mixing loss behind impeller

$h_{i f} \quad$ skin friction loss in impeller

$h_{V} \quad$ total hydraulic loss in volute

$h_{V d e} \quad$ expansion loss in nozzle

$h_{\text {Vdf }} \quad$ skin friction loss in nozzle

$h_{V f} \quad$ skin friction loss in spiral body of volute

$H$ pump head

$H_{t} \quad$ theoretical head of impeller

$H_{W} \quad$ head of duty point for water

$K_{Q} \quad$ correction factor of flow rate to viscosity

$k_{s} \quad$ roughness of wetted wall

$L_{i} \quad$ blade length

$L_{K} \quad$ length of discharge nozzle

$L_{V} \quad$ length of spiral body of volute

$n \quad$ pump rotating speed

$n_{s} \quad$ specific speed of pump

$\begin{array}{ll} & n_{\mathrm{s}}=3.65 n \sqrt{Q} / H^{3 / 4}\left(\mathrm{r} / \mathrm{min}, \mathrm{m}^{3} / \mathrm{s}, \mathrm{m}\right) \\ P & \text { shaft-power of pump } \\ P_{d} & \text { power due to disc friction of impeller } \\ P_{h} & \text { hydraulic power of pump } \\ Q & \text { pump flow rate }\end{array}$

\section{INTRODUCTION}

The effects of blade discharge angle of impeller on the performance of centrifugal pumps have been investigated since 1950s. Kamimoto and Matsuoka (1956) experimentally investigated various model centrifugal pump impellers with six logarithmic spiral blades with the constant angles of $30^{\circ}, 50^{\circ}, 70^{\circ}$ and $90^{\circ}$ respectively by using water as working fluid. The impeller was closed type and subject to a rotting speed of $1750 \mathrm{r} / \mathrm{min}$. It was made clear that the impeller with $30^{\circ}$ discharge angle achieved best performance. The maximum variation of impeller hydraulic efficiency was as high as $40 \%$. Varley (1961) conducted the experimental investigations into the effects of the blade discharge angle on the performance of a double suction centrifugal pump with specific speed of 36 when handling water. The pump rotating speed was $1400 \mathrm{r} / \mathrm{min}$. The impeller of the pump was closed type and there were five blades in it. The blade pattern was a single arc. The inlet angle of the blade was $36^{\circ}$, the discharge angle were $15^{\circ}, 27^{\circ}, 40^{\circ}, 59^{\circ}, 74^{\circ}$ and $88^{\circ}$, respectively. The results demonstrated that the head increased with increasing discharge angle and the larger the flow rate, the more the increase in head. When the blade discharge angle was between $15^{\circ}$ and $59^{\circ}$, the maximum variation of efficiency of the pump was only about $1.6 \%$. Toyokuro et al (1979) made experiments on the influences of the discharge angle on the infinite number of blades

$\alpha \quad$ equivalent expansion angle of discharge

nozzle

$\beta_{1} \quad$ blade inlet angle

$\beta_{2} \quad$ blade discharge angle

$\delta \quad$ thickness of sub-laminar layer

$\Delta V_{u 2} \quad$ slip velocity at impeller outlet

$\eta \quad$ pump efficiency

$\eta_{h} \quad$ pump hydraulic efficiency

$\eta_{V} \quad$ pump volumetric efficiency

$\eta_{m} \quad$ pump mechanical efficiency

$\theta$ equivalent diffusion angle of impeller passage

$\lambda \quad$ skin friction coefficient

$v \quad$ kinematic viscosity of fluid

$\xi \quad$ expansion loss coefficient

$\xi_{0}$ expansion loss coefficient when $R e \geq 4 \times 10^{5}$

$\rho$ density of fluid

$\sigma \quad$ slip factor

$\mathrm{Z}$ number of blades

$\varphi_{0} \quad$ circumferential angle of tongue of volute

$\psi_{2} \quad$ blockage factor of blade at outlet

$\omega \quad$ angular speed of impeller

Abbreviation

BEP best efficiency point of pump

Subscription
i $\quad$ impeller
V $\quad$ volute

performance of a single-stage, end-suction and cantilevered centrifugal pump with specific speed 107 at rotating speed $1400 \mathrm{r} / \mathrm{min}$ when handling water and viscous oils. The impeller was also closed type with six blades and the blade pattern was single arc too. The discharge angles were $23^{\circ}$ and $65^{\circ}$ respectively. The fluid viscosity was up to $390 \mathrm{~mm}^{2} / \mathrm{s}$ (Reynolds number $R e_{2}$ was between $10^{4}$ and $1.9 \times 10^{6}$ ) in the experiments.

The results revealed that the head was improved as the discharge angle increased, the larger the flow rate was, and the more the head was improved. However, the efficiency of the pump for the discharge angle of $65^{\circ}$ was just about $1 \%$ less than that of $23^{\circ}$ when pumping water. Not only did the head increase with increasing discharge angle, but the efficiency was improved also by $8 \%$ while handling viscous oils. The higher the oil viscosity, the larger the improvement in efficiency. Tanaka and Ohashi (1984) made experiments on a series of semi-open, end-suction centrifugal pump impellers while transporting highly viscous oils with viscosity of $1-1500 \mathrm{~mm}^{2} / \mathrm{s}$ (Reynolds number $R e_{2}$ was in the range of $\left.4 \times 10^{2}-1.0 \times 10^{6}\right)$. The radial blades $\left(90^{\circ}\right)$ could achieve best performance for high viscosity liquids. Aoki et al (1985) and Ohta et al (1990, 1996) experimentally studied the influences of the discharge angle on the performance of a single-stage, end-suction and cantilevered centrifugal pump with the specific speed of 70 when handling water and viscous oils. The fluid viscosity was in the range of $1-650 \mathrm{~mm}^{2} / \mathrm{s}$ 
(Reynolds number $R e_{2}$ was in $1.5 \times 10^{4}-2.5 \times 10^{6}$ ). The impeller was closed or semi-open type with six blades. The two-arc-shaped blade exit angles were $10^{\circ}, 20^{\circ}$, $40^{\circ}, 60^{\circ}, 73^{\circ}$, respectively. The experimental results were similar to those of Toyokuro et al (1979).

In summary so far, when the centrifugal pumps handle water, one experiment shows the pump hydraulic efficiency can be improved by $40 \%$ with a variable blade exit angle (Kamimoto et al, 1956), but the other experiment indicates the pump efficiency just is improved by $1.6 \%$ (Varley, 1960). Obviously, this dispute needs to be confirmed experimentally. As a pump handling viscous oils, however, a consistent result seems to be achieved, i.e., the blade exit angle has substantial influence on both head and efficiency for model impellers. The results suggest large discharge angle is helpful to improve both head and efficiency for a liquid with higher viscosity than water.

The centrifugal oil pump with low specific speed has found significant applications in oilfields and refineries in China. What will happen in the performance for such a kind of pump when pumping various viscous oils for various blade discharge angles is interesting since this will result into an improved pump performance to save energy.

In this paper, a standard industrial centrifugal oil pump of type 65Y60 with low specific speed of 41.6 was employed as a test model and the effects of its blade discharge angle on the performance were investigated experimentally as the pump handled both water and viscous oils with various viscosities, respectively.

The results showed that the influence of the angle on the head was dominated at all viscosity conditions. The impeller with the discharge angle of $25^{\circ}$ illustrated a best efficiency as the viscosity of liquid was less than $200 \mathrm{~mm}^{2} / \mathrm{s}$; however, as the viscosity was more than this value, the impeller with the discharge angle of $60^{\circ}$ demonstrated highest efficiency. Moreover, a onedimensional hydraulic model was proposed to investigate the effects of blade discharge angle at various viscosities analytically.

\section{EXPERIMENTAL SETUP}

\subsection{Test Rig}

The sketch of the test rig for measuring the performance of centrifugal oil pump while handling water or viscous oil has been shown by Li (2002). The detail of the test rig can be found out in that reference. An oil temperature control system was installed in the tank of the rig in order to heat the oil and reduce its viscosity. The measurement uncertainties of flow rate, head, shaft power and efficiency were $0.707 \%, 0.205 \%, 0.515 \%$ and $0.898 \%$, respectively $(\mathrm{Li}, 2002)$.

\subsection{Pump and Blades Patterns}

The test pump was a standard industrial centrifugal oil pump of type $65 \mathrm{Y} 60$ with side-suction entry and singlestage, which has been extensively applied to transport clean crude oils and other liquid petroleum products at $40^{\circ} \mathrm{C} \sim 350^{\circ} \mathrm{C}$ in China (Fig. 1). The pump duty specifications are as follows: the flow rate $Q=25 \mathrm{~m}^{3} / \mathrm{h}$, head $H=60 \mathrm{~m}$, rotating speed $n=2950 \mathrm{r} / \mathrm{min}$, specific speed $n_{s}=41.6$. The impeller was closed type with five twisted three-dimensional blades (see Fig. 1c).

The cross-sectional drawings of the pump and impeller have been shown in Fig. 1 (a) and (b). The main dimensions of the impeller are: $D_{2}=213 \mathrm{~mm}$, $b_{2}=7.5 \mathrm{~mm}, D_{1}=62 \mathrm{~mm}, b_{1}=16 \mathrm{~mm}, \mathrm{Z}=5, \beta_{2}=25^{\circ}$, $S_{u 2}=10 \mathrm{~mm}$. The major dimensions of the volute are as follows: $D_{3}=240 \mathrm{~mm}, \quad b_{3}=16 \mathrm{~mm}, \quad D_{9}=60 \mathrm{~mm}$, $F_{8}=858 \mathrm{~mm}^{2}, L_{k}=250 \mathrm{~mm}, \varphi_{0}=36^{\circ}$.
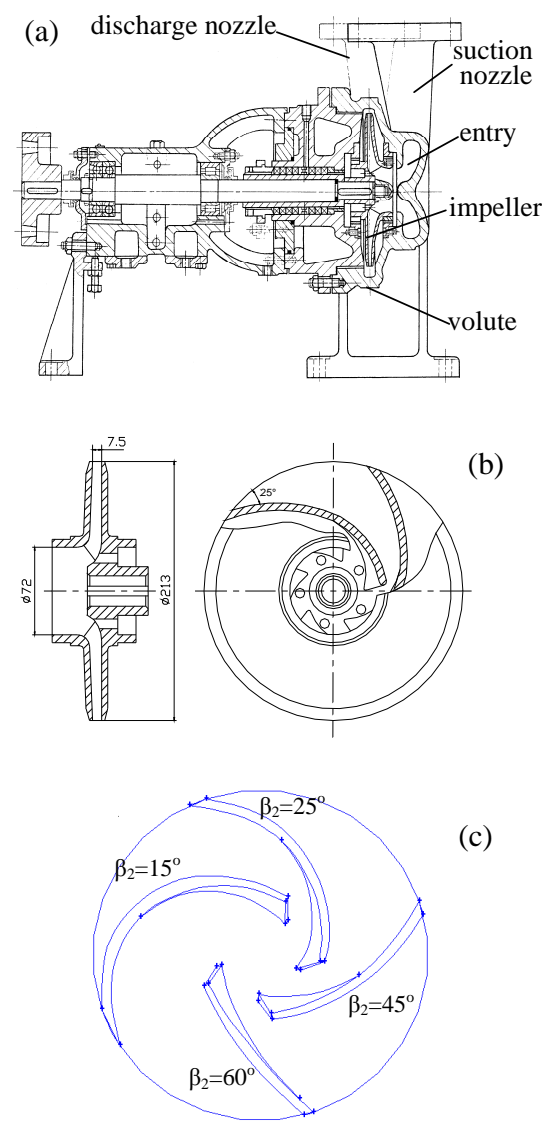

Fig. 1. Pump cross-sectional view (a), impeller style (b) and blade pattern (c)

The three impellers with discharge angle, $\beta_{2}, 15^{\circ}, 45^{\circ}$ and $60^{\circ}$, were prepared to illustrate their influence on the performance of the pump. The blade length, blade warp angle and $S_{u 2}$ were different from thoss of the original impeller with a discharge angle of $25^{\circ}$, but the rest geometrical dimensions remained unchanged.

The number of blades of those impellers was five and the blade patterns were NURBS curves. The comparison of the four blade patterns has been demonstrated in Fig.1 (c). 
W.G. Li / JAFM, Vol. 4, No. 2, Issue 1, pp. 105-119, 2011.
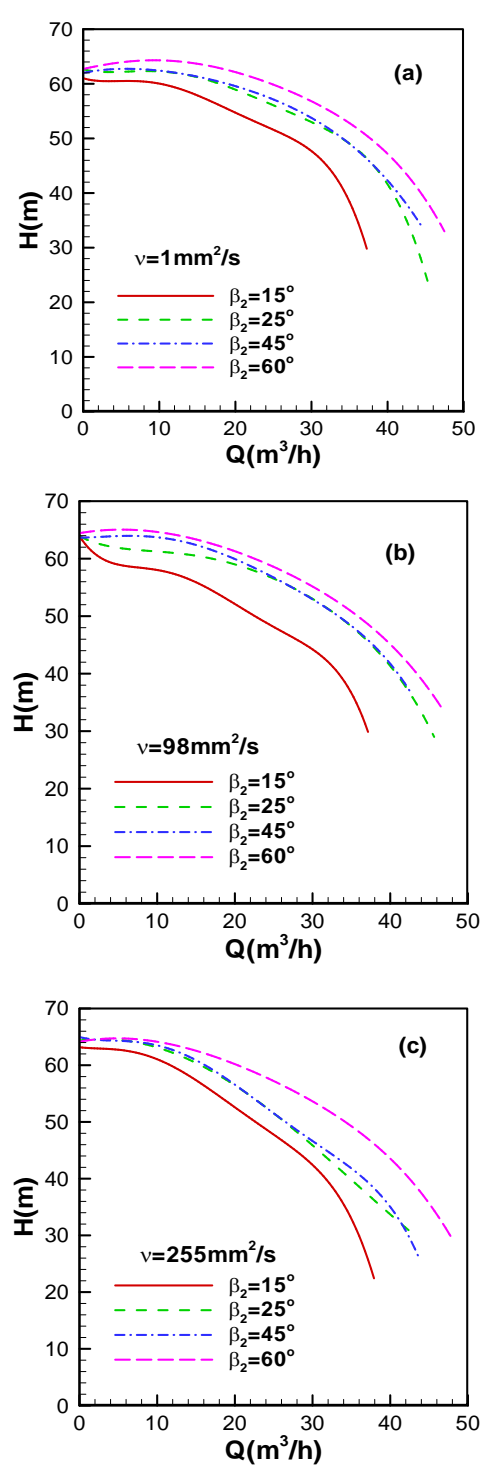

Fig. 2. Head curves in terms of flow rate at three viscosity values

\subsection{Working Liquids}

The working liquids were both water and China 100\# machine oil in the performance experiments, respectively. It was made clear that both liquids are Newtonian fluid by using a rotating dynamic viscosity meter. The density and kinematical viscosity of water are $1000 \mathrm{~kg} / \mathrm{m}^{3}, 1 \mathrm{~mm}^{2} / \mathrm{s}$ at $20^{\circ} \mathrm{C}$, respectively. However, the density and dynamic as well as kinematical viscosities of the oil demonstrated a variation with temperature in the experiments when the temperature was varied in the range of $15^{\circ} \mathrm{C}-65^{\circ} \mathrm{C}$. Subsequently, based on the test data, the formula for calculating the density and kinematical viscosity of the oil was got and read as

$\left\{\begin{array}{l}\rho=906.653-0.5267 T \\ v=\frac{1000}{1.0112 \times 10^{-2} T^{2}-0.2222 T+2.8658}\end{array}\right.$

The values of the kinematical viscosity of working liquids, namely 1 (water), 29, 45, 75, 98, 134, 188 and
$255 \mathrm{~mm}^{2} / \mathrm{s}$ (oil), were chosen in the experiments. The performance tests were conducted in such a way that the oil temperature was raised from low to high in order to control its viscosity conveniently.

The oil temperature was monitored both in the oil tank and in the pump suction nozzle. Usually, the oil temperature rises less than $2^{\circ} \mathrm{C}$ during a performance test. In those experiments, Reynolds number $R e_{2}$ was in the range of $1.37 \times 10^{4}-3.5 \times 10^{6}$.

\section{EXPERIMENTAL RESULTS}

\subsection{Performance Curves}

Figure 2 shows the variation of head curve versus flow rate when the viscosities of working liquid are 1,98 and $255 \mathrm{~mm}^{2} / \mathrm{s}$, respectively. A larger discharge angle causes the pump to develop a higher head than a smaller one does. As the viscosity is $1 \mathrm{~mm}^{2} / \mathrm{s}$, the peak head has occurred at a flow rate of $Q=8 \mathrm{~m}^{3} / \mathrm{h}$; rather than at zero flow rate.
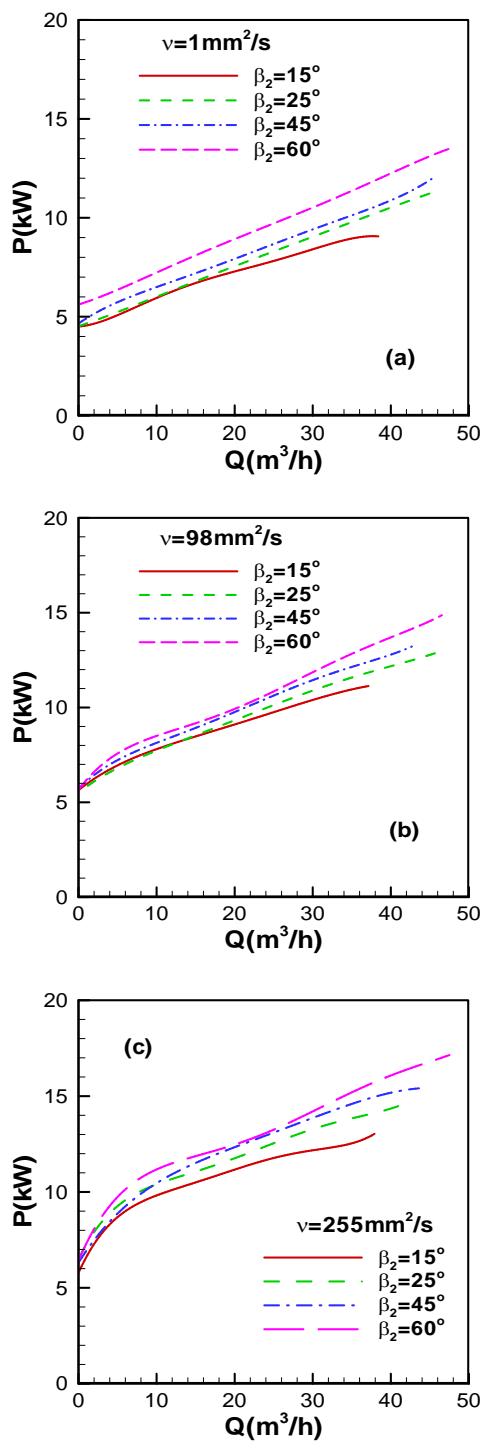

Fig. 3 Shaft-power curves in terms of flow rate at three viscosity values 
This is so-called unstable or hooked shape head curve effect. Fortunately, such an unstable curve disappears gradually with increasing viscosity. No matter whatever value the viscosity is, the maximal change of the head curve due to the discharge angle variation almost maintains the same amount, i.e., the effect of discharge angle on the head curve seems to be independent upon the viscosity of the working liquid. Hence, changing discharge angle should be an effective way for altering the head of centrifugal oil pump.

Figure 3 illustrates the variation of shaft power curves in terms of flow rate as the viscosities of working liquid are 1,98 and $255 \mathrm{~mm}^{2} / \mathrm{s}$. Like the head curves, the effect of the discharge angle on the shaft-power curve appears to be independent on the viscosity of the working liquid. Further, a larger discharge angle definitely consumes more power than a smaller one does.

Figure 4 demonstrates the variation of the efficiency curves against flow rate while the viscosities of working liquid are 1,98 and $255 \mathrm{~mm}^{2} / \mathrm{s}$. First of all, the efficiency reduces continuously with increase in viscosity.

Unlike those in the head curves, the effect of the discharge angle on the efficiency curve is dependent on the viscosity of the working liquid. As the viscosity is less than $200 \mathrm{~mm}^{2} / \mathrm{s}$, the efficiency of the pump with a discharge angle of $25^{\circ}$ is higher than that with an angle of $60^{\circ}$. However, for the viscosity more than $200 \mathrm{~mm}^{2} / \mathrm{s}$, the situation is reversed.

\subsection{Hydraulic Parameters at BEP}

In order to illustrate the effect of viscosity on the performance of the centrifugal oil pump with various discharge angles more clearly, Fig. 5 is applied to show the hydraulic parameters, such as head, hydraulic power and efficiency at the best efficiency point (BEP) against viscosity. The impeller with a discharge angle of $60^{\circ}$ always develops a higher head than the others at the BEP. As the viscosity is higher than $200 \mathrm{~mm}^{2} / \mathrm{s}$, the head is improved by $10 \%$; as the viscosity is up to $255 \mathrm{~mm}^{2} / \mathrm{s}$, the efficiency is increased by $3 \%$ compared to the original impeller with $25^{\circ}$ exit angle. However, at the high flow rates, the efficiency is improved by $6 \%$. The reason for this is that the hydraulic power of the impeller with $60^{\circ}$ discharge angle drops off very slowly than the others with increasing viscosity. A similar behavior was observed in the pervious studies (Toyokuro et al, 1979; Tanaka and Ohashi, 1984; Ohta and Aoki, 1990). This fact seems to reveal that the blade exit angle effect on the pump head and efficiency is nearly equal. When a pump pumping water, it was shown experimentally that the blade exit angle effect was dominated on the pump efficiency (Kamimoto and Matsuoka, 1956) rather than on the head. However, the result was discovered that the effect on the head not efficiency was substantial (Varley, 1961). Our current results don't agree with them.
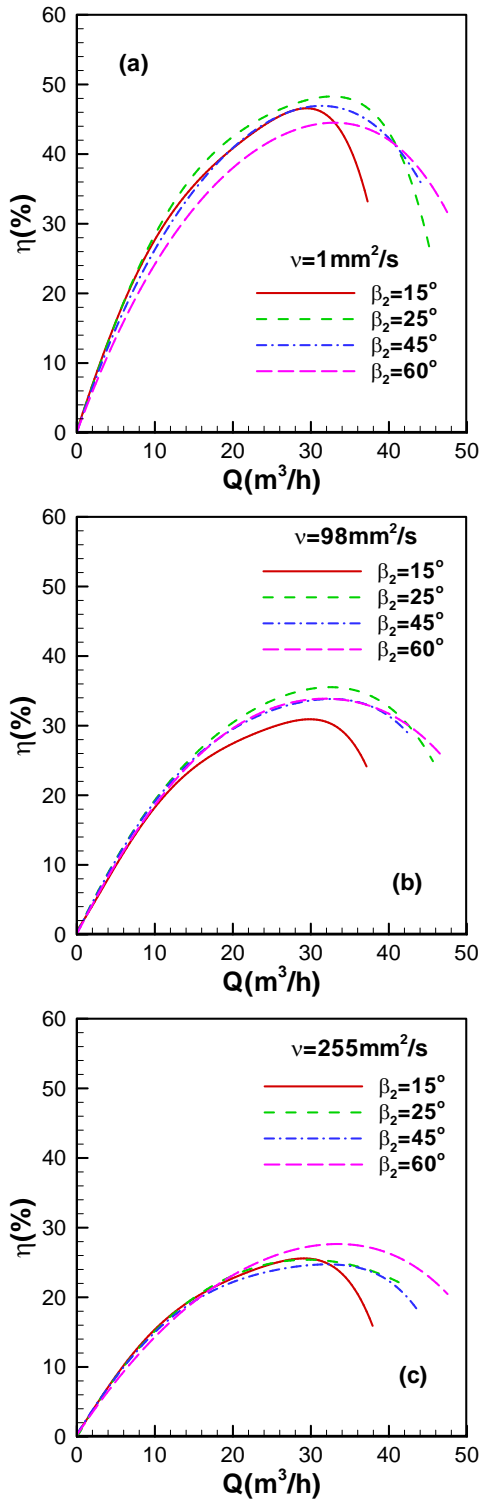

Fig. 4 Efficiency curves against flow rate at three viscosity values

From the point of view of efficiency, while the viscosity of liquid is lower than $200 \mathrm{~mm}^{2} / \mathrm{s}$, the performance of the original impeller with a discharge angle of $25^{\circ}$, is best. However, while the viscosity is higher than this value, the performance of the newly designed impeller with a discharge angle of $60^{\circ}$ is best. The impeller with an exit angle less than $25^{\circ}$ has a very poor performance.

\subsection{Slip Factor}

The slip factor is used to specify the flow slip effect at the exit of a centrifugal pump impeller and is a key parameter to establish a theoretical head developed by the impeller. There are several versions of definition for the slip factor (Qiu, Mallikarachchi and Anderson, 2007). 

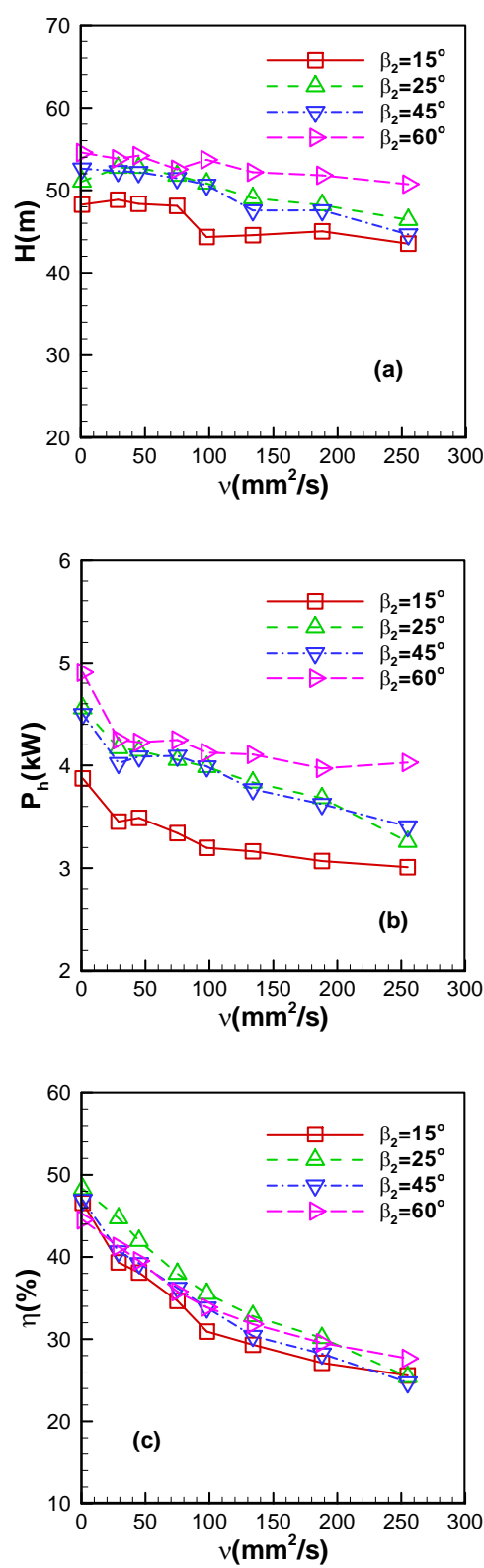

Fig. 5 Head (a), hydraulic power (b) and efficiency (c) at BEP in terms of viscosity

However, the following definition (Brennen, 1994) is adopted in this paper

$\sigma=\frac{u_{2}-\Delta V_{u 2}}{u_{2}}=1-\frac{\Delta V_{u 2}}{u_{2}}$

where the slip velocity of fluid (Fig. 6) depends on the impeller geometry and flow rate as well as fluid viscosity etc. Traditionally, the slip factor of a centrifugal pump impeller is frequently estimated by means of the Weisner's or Stodola's formulas. The Weisner's expression is read as (Brennen, 1994)

$\sigma=1-\frac{\sqrt{\sin \beta_{2}}}{Z^{0.7}}$

and the Stodola's relation is written as (Brennen, 1994) $\sigma=1-\frac{\pi \sin \beta_{2}}{Z}$

These equations do not involve the effects of both fluid viscosity and flow rate.

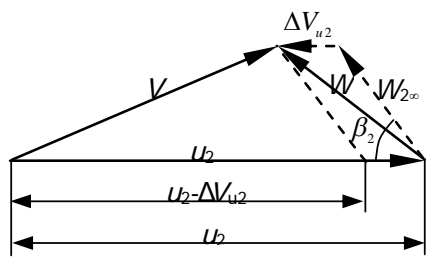

Fig. 6 Velocity triangle at impeller exit

It is interesting to note that a slip factor of centrifugal pump impeller can be calculated based on the pump experimental performance curve and impeller geometry ( $\mathrm{Li}, 2004)$. The following equation is used to estimate the slip factor at the BEP

$\sigma=\frac{g}{2 u_{2}^{2}}\left[2 H-Q\left(\frac{d H}{d Q}-\frac{u_{2}}{g \eta_{v} F_{2} \tan \beta_{2}}\right)\right]$

where the volumetric efficiency, $\eta_{V}$ was calculated by using Stepanoff's method for the geometrical parameters of both the wear-rings installed in the impeller shroud and hub, ring radial clearance as well as the pressure differential across the rings (Stepanoff, 1948). The details of the calculation are available in $\mathrm{Li}$ (2004).

Figure 7 illustrates the slip factor at the BEP as a function of blade discharge angle at the viscosities of 1 , 98 and $255 \mathrm{~mm}^{2} / \mathrm{s}$. From Fig. 7, it was learnt that no matter whatever value the viscosity is, the slip factors calculated by using the experimental head-flow rate relations decrease with increasing blade discharge angle. The fluid viscosity does affect the slip factor, but the effect is not substantial. These findings are consistent with those illustrated by Toyokuro et al (1979) and Ohta (1999).

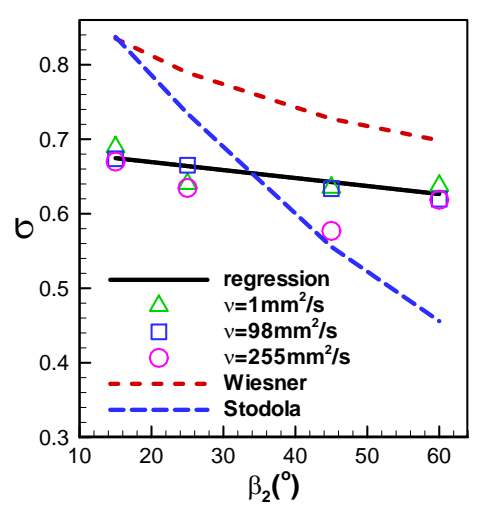

Fig. 7 Slip factor as a function of blade exit angle at three viscosity values at BEP

If regardless of the viscosity effect, the scattered points of the slip factors estimated via the experimental data can be represented by the following linear equation with respect to the blade discharge angle 


$$
\sigma=0.690689-0.00106984 \beta_{2}
$$

Moreover, the slip factors established by the Weisner's and Stodola's formulae show extremely large difference from those determined by the experimental data. This suggests that if those expressions are applied to estimate the slip factors for highly viscous oils, significant errors will be caused.

\section{Model Exit ANgle EFfECT}

The centrifugal pump performance prediction was traditionally based on the hydraulic loss analysis in a pump (Aisenstein, 1927; Pigott, 1945; Rathod \& Donovan, 1980; Takagi et al, 1980; Stirling, 1982; Aly \& Al-Zubaidy, 1992; Yoon et al, 1998; Oh \& Chung, 1999; Zaher, 2001). In those predictions, the fluid viscosity remains unchanged. More recently, the effect of fluid viscosity on centrifugal pump efficiency was shown analytically with the scaling laws related to the pump mechanical, volumetric and hydraulic efficiencies (Gulich, 1999a; 1999b; 2003). The performance of three centrifugal pumps was evaluated by considering blade loading loss and impeller and volute friction loss based on pipe flow at a variety of fluid viscosity (Hamkins and Hergt, 1987). Unfortunately, any useful equations for estimating those losses were not presented by them at all. The hydraulic losses in centrifugal oil pumps were calculated by using the boundary layer theory in fluid mechanics at different viscosities of liquid pumped to explore the cause for the "suddenrising head" effect in the pumps ( $\mathrm{Li}, 2000)$. However, in the calculation the diffusion loss in the impellers was ignored.

In this section, it is intended to theoretically investigate the effect of exit blade angle on the pump performance at different viscosities of fluid at the pump duty point and to compare with the experimental observations. In fact, such an investigation is equivalent to how to exactly estimate hydraulic losses inside the pump, eventually causing precise predictions of the performance parameters at those viscosities.

\subsection{Hydraulic Loss Model}

It is assumed the flow in the pump is one-dimensional, steady, laminar or turbulent. Since the fluid velocity in the side-entry of the pump shown in Fig. 1 (a) is much lower than those in the impeller and volute, the hydraulic losses can be ignored in the entry. Just the losses across the impeller and volute are considered here. It is believed that the angle of attack to the entrance of blade is so small that the shock loss there also can be neglected. Therefore, a flow just suffers from skin friction and diffusion losses in an impeller. For the volute, a flow is subject to the skin friction loss in the spiral body as well as skin friction loss and diffusion in the discharge nozzle. A mixing loss occurs at the boundary between the impeller exit and volute inlet.

For convenience, the complicated flow passages in the impeller and volute are converted into straight circular pipes by using hydraulic diameter properly; the skin friction coefficients for such pipes are applied to determine the friction loss in the actual flow passages.

The experimental centrifugal oil pump is a low specific speed pump because the specific speed of its impellers is just around 41. In the impellers of such a pump, the expansion of the cross-sectional area of flow channels is much large in the radial direction even a slight contraction in the axial direction. Consequently, the impellers are subject to a diffusion loss, which must be taken into account in the hydraulic loss model.

\subsection{Hydraulic Loss in Impeller}

The major dimensions of the impeller and volute were sketched in Fig. 8. The hydraulic losses in the impeller consist of the skin friction and diffusion losses.
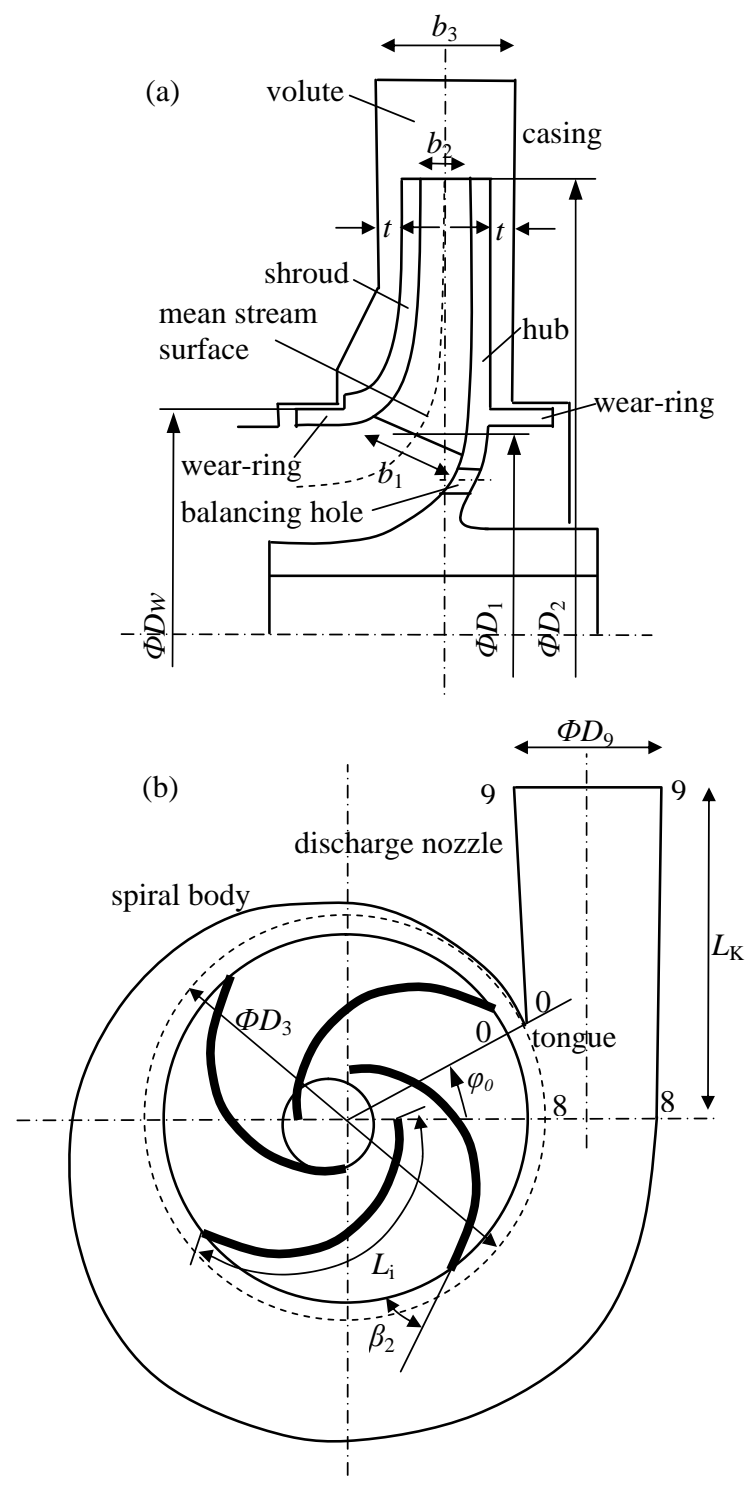

Fig. 8 Major dimensions of impeller (a) and volute (b)

The following expression is employed to figure out the friction loss

$h_{i f}=\lambda \frac{L_{i}}{D_{h i}} \frac{W^{2}}{2 g}$ 
where the blade length $L_{i}$ on the mean stream-surface was correlated to the blade exit angle $\beta_{2}$ of four experimental impellers by the following equation

$L_{i}=0.0033 \beta_{2}^{2}-0.4567 \beta_{2}+27.5$

The mean relative velocity of fluid through the impeller passages $W$ is equal to half sum of those at the blade inlet and outlet, respectively

$W=\left(W_{1}+W_{2}\right) / 2$

The hydraulic diameter $D_{h i}$ is estimated by the formula

$D_{h i}=\frac{1}{2}\left(\frac{\frac{4 \pi D_{1} b_{1}}{Z}}{2 b_{1}+\frac{2 \pi D_{1}}{Z}}+\frac{\frac{4 \pi D_{2} b_{2}}{Z}}{2 b_{2}+\frac{2 \pi D_{2}}{Z}}\right)$

Further, the equation can be simplified as follows

$D_{h i}=\frac{D_{1} b}{Z b_{1}+\pi D_{1}}+\frac{D_{2} b_{2}}{Z b_{2}+\pi D_{2}}$

The skin friction coefficient, $\lambda$, depends on the flow regime in the passages and Reynolds number $R e_{i}$ $\left(R e_{i}=W D_{h i} / v\right)$ and the roughness of wetted surfaces , $k_{s}$.

The diffusion loss in the impellers can be calculated by using the following equation

$h_{i d}=\xi \frac{W_{1}^{2}}{2 g}$

The diffusion coefficient $\xi$ is dependent on the equivalent expansion angle of the impeller flow passage $\theta$, which is determined by

$\theta=2 \tan ^{-1}\left[\frac{\pi\left(D_{2}-D_{1}\right)}{2 Z L_{i}}\right]$

For the pervious experimental impellers, the equivalent expansion angle can be expressed in terms of blade discharge angle

$\theta=-0.0001 \beta_{2}^{3}+0.0099 \beta_{2}^{2}+0.1262 \beta_{2}+19.6618$

\subsection{Hydraulic Loss in Volute}

There are three kinds of hydraulic loss in the volute. One is the skin friction loss in the spiral body, one is the friction and diffusion losses in the discharge nozzle and one is the mixing loss behind the impeller.

The friction loss along the wall of the spiral body can be calculated by using the following equation

$h_{V f}=\lambda \frac{L_{V}}{D_{h V}} \frac{V_{3}^{2}}{2 g}$

The hydraulic diameter $D_{h V}$ is related to the mean cross-section area of the volute as follows

$D_{h V}=\frac{4 F_{m}}{b_{3}+2 F_{m} / b_{3}}$
The mean cross-section area is decided by the areas of the cross-sections $0-0, F_{0}$, and $8-8, F_{8}$ as follows

$F_{m}=\frac{1}{2}\left(F_{0}+F_{8}\right)$

Note that the area $F_{0}=0.5\left(D_{3}-D_{2}\right) b_{3}$ and $F_{8}$ has included the area of $0.5\left(D_{3}-D_{2}\right) b_{3}$.

The mean velocity of fluid, $V_{3}$, in the volute is simply estimated via

$V_{3}=Q\left(1-\varphi_{0} / 360\right) / F_{8}$

The length of the spiral body, $L_{V}$, is determined by using the expression

$L_{V}=\pi D_{3}\left(1-\varphi_{0} / 360\right)$

Likewise, the skin friction coefficient, $\lambda$, also depends on the flow regime in the volute and Reynolds number $R e_{V}\left(R e_{V}=V_{3} D_{h V} / v\right)$ and the roughness of wet surfaces , $k_{s}$.

The skin friction loss in the discharge nozzle is estimated by means of the following expression

$h_{v d f}=\lambda \frac{L_{k}}{D_{89}} \frac{V_{89}^{2}}{2 g}$

The mean diameter of the discharge nozzle can be determined by

$D_{89}=\frac{1}{2}\left(D_{8}+D_{9}\right)$

The equivalent diameter of the section $8-8$ is given by

$D_{8}=\sqrt{\frac{4 F_{8}}{\pi}}$

The velocity of fluid through the pipe with a diameter of $D_{89}$ is calculated by the equation

$V_{89}=Q / F_{89}$

where the mean area $F_{89}=\pi D_{89}^{2} / 4$. The skin friction coefficient, $\lambda$, is in terms of the Reynolds number $\operatorname{Re}_{d}\left(\operatorname{Re}_{d}=V_{89} D_{89} / v\right)$ and the roughness of wet surfaces , $k_{s}$.

The diffusion loss in the discharge nozzle is determined by the following equation

$h_{\text {Vde }}=\xi \frac{V_{3}^{2}}{2 g}$

The diffusion loss coefficient $\xi$ depends on the equivalent expansion angle of the discharge nozzle, $\alpha$, is written as

$\tan \frac{\alpha}{2}=\frac{D_{9}-D_{8}}{2 L_{K}}$

The mixing loss behind an impeller is considered to be the loss due to a sudden expansion of the meridian flow and the loss due to turbulent or laminar shearing effect 
in the tangential direction between flow exiting the impeller and that in the volute. The mixing loss behind the impeller is calculated by the equation

$h_{i e}=\frac{\left[1-b_{2} / b_{3}\right]^{2} V_{m 2}^{2}+\left(V_{u 2}-V_{3}\right)^{2}}{2 g}$

The first term is Eq. (26) is according to the sudden expansion loss in White (1994), but the second term is proposed by the author of this paper.

\subsection{Hydraulic Parameter Estimate}

For the volumetric efficiency of a pump, $\eta_{V}$, is slightly increased by a higher viscosity of liquid pumped (Kurokawa, 1990). Since its variation is very small, it is simply be estimated by using the Lomakin's empirical equation (Kurokawa, 1990)

$\eta_{v}=\frac{1}{1+0.68 n_{s}^{2 / 3}}-0.07$

Note that the second term is added into Eq. (27) by the author of this paper to approximately account for the effect of the wear-ring and balancing holes in the experimental impeller hubs on the volumetric efficiency.

The hydraulic efficiency of the pump, $\eta_{h}$ can be determined according to the theoretical head and total hydraulic losses in the impeller and volute. It is expressed by

$\eta_{h}=1-\frac{h_{l}}{H_{t}}$

The total hydraulic loss, $h_{l}$, is the sum of all the losses in the impeller and volute, it is read as

$h_{l}=h_{i f}+h_{i d}+h_{i e}+h_{V f}+h_{V d f}+h_{V d e}$

The theoretical head generate by the impeller, $H_{t}$, depends on the flow rate and impeller geometry as well as rotating speed. It is specified by the Euler equation for turbomachine

$$
H_{t}=\frac{u_{2}}{g}\left(\sigma u_{2}-\frac{Q}{\eta_{V} \pi D_{2} b_{2} \psi_{2} \tan \beta_{2}}\right)
$$

The slip factor has been decided with Eq. (6). The blade blockage coefficient at the outlet, $\psi_{2}$, is calculated via the expression

$\psi_{2}=1-Z S_{u 2} / \pi D_{2}$

The mechanical efficiency of the pump is estimated by using the mechanical loss and shaft-power as follows

$\eta_{m}=1-\frac{1.5 P_{d}}{P}$

It was found that the measured disk friction losses in the actual impellers usually were higher than those estimated with empirical equations (Nemdili and Hellmann, 2007). The factor 1.5 in Eq. (32) is used to take this effect into account. The disk friction power loss of the impeller, $P_{d}$, is calculated by the following equation (Gulich, 1999a)
$P_{d}=\frac{1}{2} C_{M} f_{g e o} \rho \omega^{3}\left(R_{2}^{5}-R_{w}^{5}\right)$

where $f_{\text {geo }}$ is the shape factor for closed type impellers of centrifugal pump, $f_{\text {geo }}=1.21$ (Gulich,1999a). The torque coefficient due to disk friction on the impeller shroud and hub, $C_{M}$ is written as (Schlichting, 1968)

$C_{M}= \begin{cases}2 \pi\left(R_{2} / t\right)\left(1 / R e_{2}\right) & R e_{2} \leq 4 \times 10^{4} \\ 2.67 / R e_{2}^{0.5} & R e_{2} \leq 3 \times 10^{5} \\ 0.0622 / R e_{2}^{0.2} & R e_{2}>3 \times 10^{5}\end{cases}$

The disk Reynolds number based on the impeller radius is defined by $R e_{2}=D_{2}^{2} \omega / 4 \mathrm{v}$. The shaft-power that a pump consumed is expressed by

$P=\rho g Q_{t} H_{t}+1.5 P_{d}$

The theoretical flow rate through an impeller is $Q_{t}=Q / \eta_{V}$. The gross efficiency of the pump can be estimated by means of the volumetric, hydraulic and mechanical efficiencies available via

$\eta=\eta_{h} \eta_{v} \eta_{m}$

At the same time, the actual head generated by the pump and the flow rare through the pump can be predicted by using the following equations accordingly. The head is read as

$H=H_{t}-h_{t}$

And the flow rate

$Q=K_{Q} Q_{W}$

The flow rate of pumped water at the design duty $Q_{W}$ is specified. However, it will get small with increasing viscosity of fluid because of viscous effect. The correction factor for such an effect is correlated to the viscosity by an equation $K_{o}=1.1051 \times 10^{-8} v^{3}-5.6347 v^{2}$ $+8.3088 \times 10^{-5} v+0.9888$.

\subsection{Friction and Diffusion Loss Coefficients}

When the Reynolds number $R e \leq 2300$, the flow in a straight duct with circular cross-section is in the laminar regime, and the skin friction coefficient is determined by the following formula (Schlichting, 1968)

$\lambda=\frac{64}{R e}$

For the impeller, $R e=R e_{i}$; for the volute, $R e=R e_{V}$; but for the discharge nozzle, $R e=R e_{d}$. When $R e>2300$, the flow in the impeller or volute is turbulent. If $k_{s} / \delta \leq 1$, then the flow is in the turbulent smooth regime. In this case, the friction coefficient $\lambda$ is independent of the relative roughness $k_{s} / D_{h}\left(k_{s} / D_{h i}\right.$ or $k_{s} / D_{h V}$ or $\left.k_{s} / D_{89}\right)$, but correlated to the Reynolds number $R e\left(R e_{i}\right.$ or $R e_{V}$ or $\left.R e_{d}\right)$. The corresponding friction loss coefficient is written as (Schlichting, 1968) 
W.G. Li / JAFM, Vol. 4, No. 2, Issue 1, pp. 105-119, 2011.

$$
\frac{1}{\sqrt{\lambda}}=2.0 \lg (\operatorname{Re} \sqrt{\lambda})-0.8
$$

The roughness of wet surfaces is $k_{s}=6 R a$ for cast walls. For the cast impeller and volute in the paper, the roughness height, which is rated as the arithmetic average deviation of the surface valleys and peaks, is $R a=40 \mu \mathrm{m}$. The thickness of the sub-laminar layer is expressed by $\delta=14.1\left(D_{h} / \operatorname{Re} \sqrt{\lambda}\right)$.

If $1<k_{s} / \delta \leq 14$, then the flow is in the turbulence transition zone. Consequently, the friction loss coefficient $\lambda$ depends on both $R e$ and $k_{s} / D_{h}$, and is read as (Schlichting, 1968)

$$
\frac{1}{\sqrt{\lambda}}=1.74-2 \lg \left(\frac{2 k_{s}}{D_{h}}+\frac{18.7}{\operatorname{Re} \sqrt{\lambda}}\right)
$$

However, if $k_{s} / \delta>14$, then the flow is in the complete turbulence rough zone, causing the coefficient $\lambda$ just related to $k_{s} / D_{h}$ and shown as follows (Schlichting, 1968)

$$
\lambda=\frac{1}{\left(1.74-2 \lg \frac{2 k_{s}}{D_{h}}\right)^{2}}
$$

The impeller and volute discharge nozzle are subject to a diffusion loss. A coefficient related to such a diffusion loss is considered to be equal to that in conical diffusers approximately. Through fitting the curves of diffusion loss coefficient in a conical diffuser with fully developed inlet flow, an empirical relation is obtained to estimate such a coefficient for any diffusion angle (White, 1994)

$$
\xi= \begin{cases}\xi_{0} & R e \geq 4 \times 10^{5} \\ \xi_{0}+0.0131 \ln \left(4 \times 10^{5} / R e\right) & R e<4 \times 10^{5}\end{cases}
$$

where $\xi_{0}=-2 \times 10^{-7} \theta^{4}+4 \times 10^{-5} \theta^{3}-2.9 \times 10^{-3} \theta^{2}+0.1096 \theta-$ 0.586 . The last term in the second expression in Eq. (43) is proposed by the author of this paper to involve low Reynolds number effect on the diffusion loss coefficient based on the experimental data presented in Fried and Idelchik (1989). For the volute discharge nozzle, angle $\theta$ should be replaced with the nozzle equivalent expansion angle $\alpha$.

\subsection{Computed Results}

The computations were conducted at the duty point $Q_{W}=25 \mathrm{~m}^{3} / \mathrm{h}$ for the density $\rho=1000$ (water), 892.96, $890.85,888.22,885.58,882.95,877.16,870.84$ (oil) $\mathrm{kg} / \mathrm{m}^{3}$ and $v=1$ (water), 29, 45, 75, 98, 134, 188 and $255 \mathrm{~mm}^{2} / \mathrm{s}$ (oil), respectively.

The blade discharge angle was varied between $15^{\circ}$ and $60^{\circ}$. The results for just the viscosities of $1,45,188$ and $255 \mathrm{~mm}^{2} / \mathrm{s}$ are shown to get tidy plots. The head and shaft-power and total efficiency in terms of blade exit angle are illustrated in Fig. 9 for different viscosities at the duty point. They can be compared with the experimental data in Fig. 10. The head estimated rises with increasing blade exit angle but reduces steadily with increasing viscosity. Note that a more change of head happens when the exit angle is between $15^{\circ}$ and $35^{\circ}$. Such variation trend seems to agree with experiments.
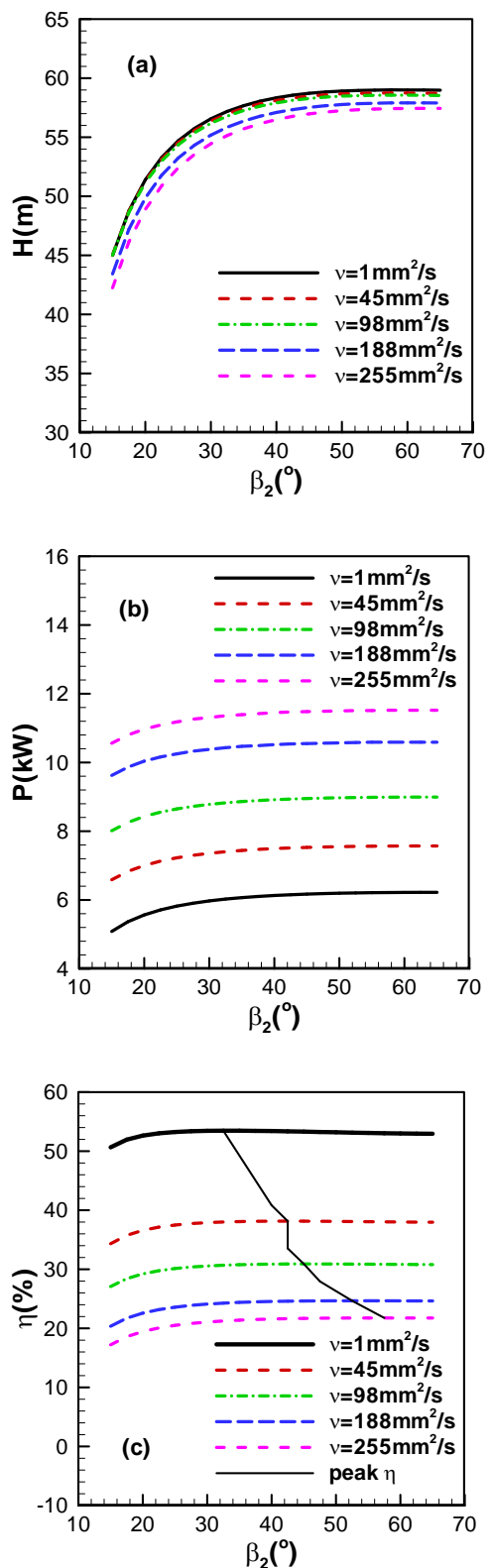

Fig. 9 Computed head (a), shaft-power (b) and efficiency (c) at duty point in terms of blade exit angle

The computed shaft-power gets large with increasing viscosity and blade discharge angle simultaneously. However, it really has a smaller rising slope than the experimental curves.

The reason for this might be Eq. (34) is unable exactly to account for the disk friction over the actual impellers with complicated shroud and hub geometry. This problem needs to be confirmed with experiment or CFD simulation. 

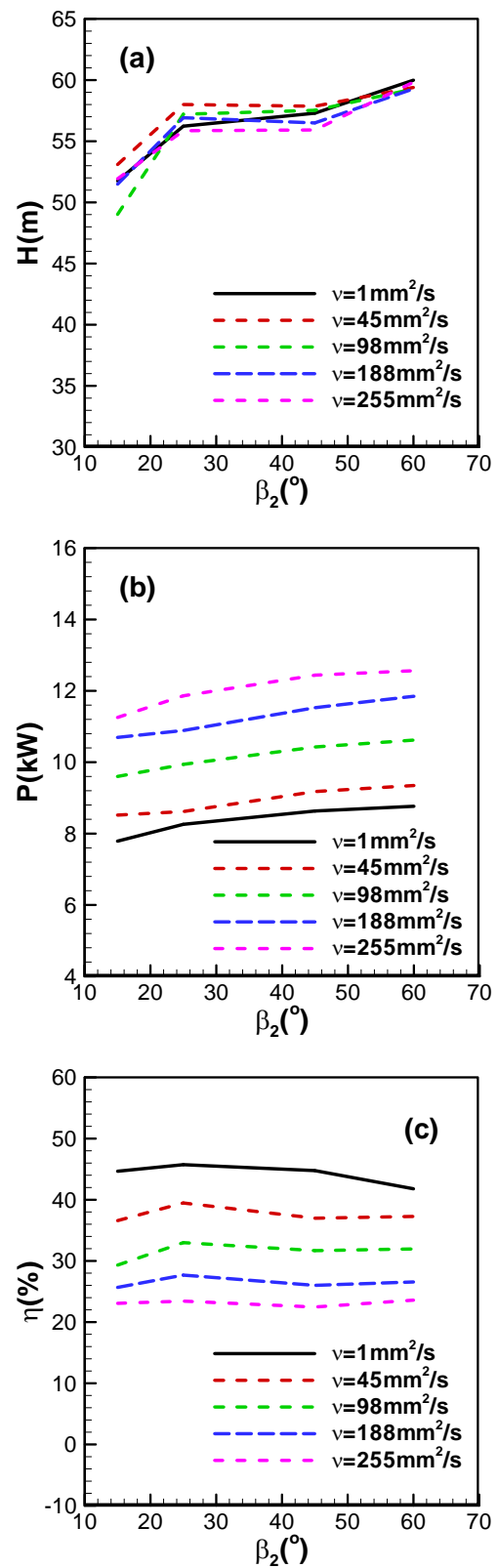

Fig. 10 Experimental head (a), shaft power (b) and efficiency (c) at duty point in terms of blade exit angle

Like the experimental pump efficiency, the estimated one is highly viscosity-dependent. Moreover, the optimal blade exit angle for best efficiency occurs at around $30^{\circ}$ at less viscosity, but with increasing of viscosity it prefers to be large one. This feature has been demonstrated in Fig. 9 clearly by using the peak efficiency curve. Note that for the experimental pump efficiency in Fig. 10, maximum efficiency is obtained at $\beta_{2}=25^{\circ}$ for $v \leq 180 \mathrm{~mm}^{2} / \mathrm{s}$ and for $v=255 \mathrm{~mm}^{2} / \mathrm{s}$ the peak efficiency is achieved at $\beta_{2}=60^{\circ}$.

The hydraulic efficiency is shown in Fig. 11 (a) for various viscosities in terms of blade discharge angle at the duty point. The effect of viscosity on the hydraulic efficiency much resembles to that on the pump total efficiency. The hydraulic efficiency is reduced

substantially by the increasing viscosity. However, at a low viscosity, a relative small blade angle allows the pump to have best hydraulic efficiency; contrarily, a relative large blade angle ensures the pump to achieve best performance at a higher viscosity. It is such a variation of hydraulic efficiency against blade exit angle that results into the pump total efficiency to present the profiles shown in Fig. 9 (c).
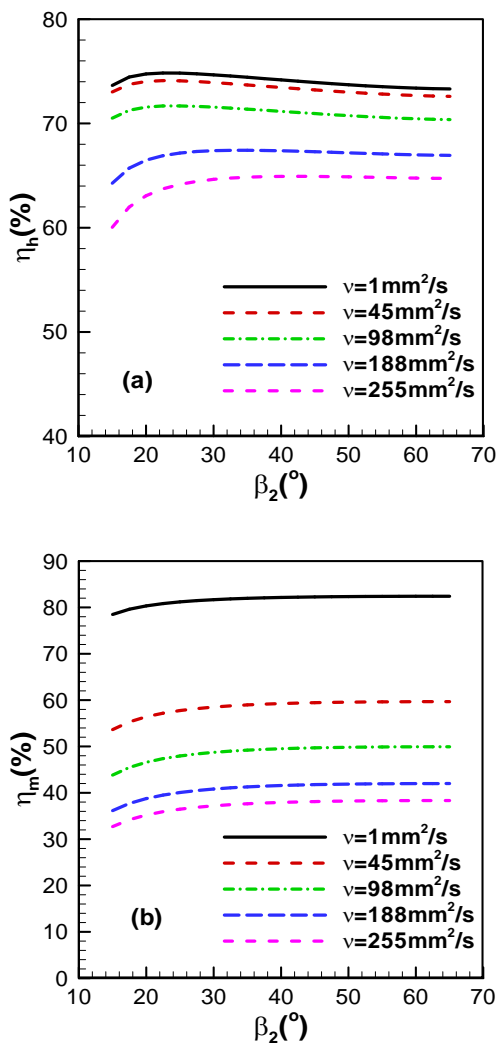

Fig. 11 Estimated hydraulic (a) and mechanical (b) efficiencies at duty point in terms of blade exit angle

The mechanical efficiency is given in Fig. 11 (b) as a function of blade discharge angle as well. It is noted that the mechanical efficiency is significantly affected by viscosity, but it is just slightly increased with increasing discharge angle.

It is the dramatic reduction in the hydraulic and mechanical efficiencies that contributes to the degraded performance of a centrifugal oil pump with increasing viscosity.

The skin friction loss in the impellers has been demonstrated in Fig. 12 (a) in terms of blade exit angle for different viscosities. This loss is very dominated in the impellers with a smaller discharge angle rather than with a larger angle at a higher viscosity. It suggests that for pumping highly viscous liquids an impeller having a larger blade angle should be more efficient compared to that with a smaller one.

The friction loss in the volute is presented in Fig. 12 (b). With increasing blade exit angle, a steady but slow increase loss is observed. This indicates an impeller 
with a larger blade angle has a negative effect on the performance of volute.
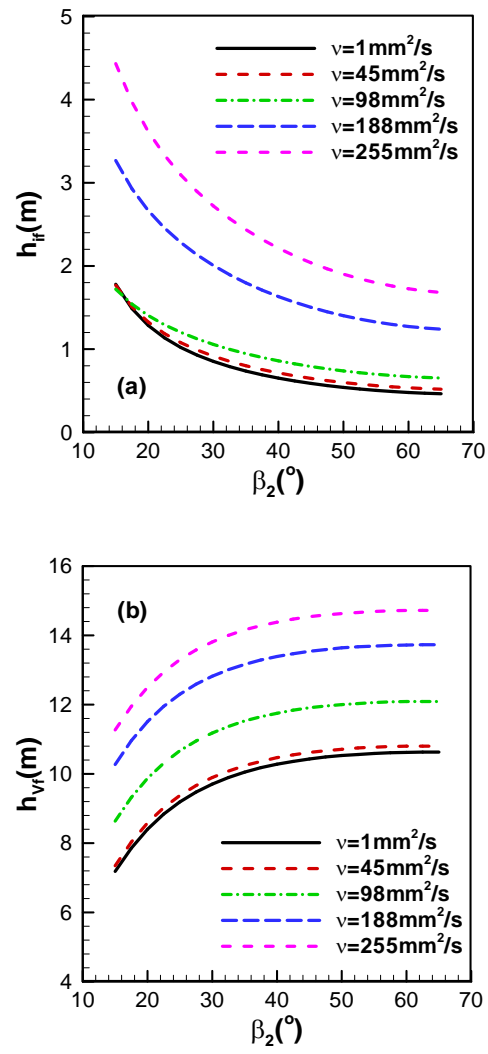

Fig. 12 Hydraulic losses in impeller (a) and volute (b) at duty point in terms of blade discharge angle

The friction loss in the impellers shows a little variation when $v \leq 98 \mathrm{~mm}^{2} / \mathrm{s}$. The small friction loss coefficients, shown in Fig. 13 (a), are responsible for this effect. The friction loss in the volute also is slightly increased when $v \leq 45 \mathrm{~mm}^{2} / \mathrm{s}$. Likewise, in that case, the small friction loss factors are seen in Fig. 13 (b).

The diffusion losses in the impeller and behind the impeller have been plotted in Fig. 14 (a) and (b), respectively, at various viscosities. The diffusion loss is slightly viscosity-dependent and gets significantly large with increasing blade exit angle. Compared to the friction loss in the impeller in Fig. 12(a), the diffusion loss is quit substantial, so that the expansion of crosssectional area of impeller passages should be carefully controlled to make the diffusion loss as small as possible. The expansion loss behind the impeller grows quickly when $\beta_{2}<40^{\circ}$, but slowly when $\beta_{2} \geq 40^{\circ}$. This loss magnitude is comparable to the skin friction loss in the impeller but much smaller than that in the volute.

The expansion loss behind the impeller consists of a sudden expansion loss in meridian plane and a mixing loss in the tangential direction. It was shown experimentally that the sudden expansion loss was independent when Reynolds number is in a range of (36) $\times 10^{4}$ for turbulent flows (Iguchi and Ohmi, 1986).
Moreover, for laminar flows, the loss wasn't changed significantly until Reynolds number less than 100 (Olveira and Pinho, 1997; Oliveira, Pinho and Schulte, 1998). In our case, since the Reynolds number is more than 420 (impeller) or 900 (volute), the sudden expansion loss can be considered to be viscosityindependent. The mixing loss may be affected by Reynolds number, but no relevant evident is found in literature, thus, it has to be treated regardless of viscosity of fluid.
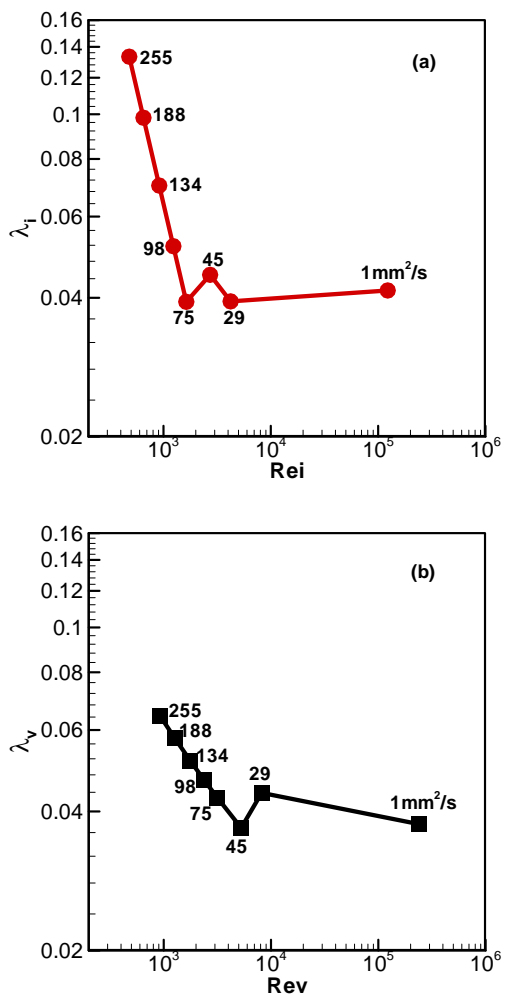

Fig. 13 Friction loss factor in terms of Reynolds number in impeller (a) with $\beta_{2}=25^{\circ}$ and volute (b), figures in plot show viscosities of fluid

The total head loss and ratio of total hydraulic loss in the impeller to that in the volute have been shown in Fig. 15 (a) and (b). The total head loss grows steadily with increasing blade exit angle and viscosity of fluid, especially when the viscosity is more than $45 \mathrm{~mm}^{2} / \mathrm{s}$. The ratio of head loss is in the range of 0.6-0.9 and depends on both blade exit angle and viscosity of fluid. This suggests that for a smaller blade exit angle the hydraulic losses in the impeller are comparable to those in the volute; however, as the blade angle increasing, the losses in the impeller are around $60 \%$ of the losses in the volute, especially when viscosity is higher than $98 \mathrm{~mm}^{2} / \mathrm{s}$. This implies the volute may play an important role in the pump performance, so that it should be designed as perfectly as possible, especially for highly viscous liquids. Note that these findings are consistent with the results presented in $\mathrm{Li}$ (2000) by using the boundary layer theory. 
W.G. Li / JAFM, Vol. 4, No. 2, Issue 1, pp. 105-119, 2011.
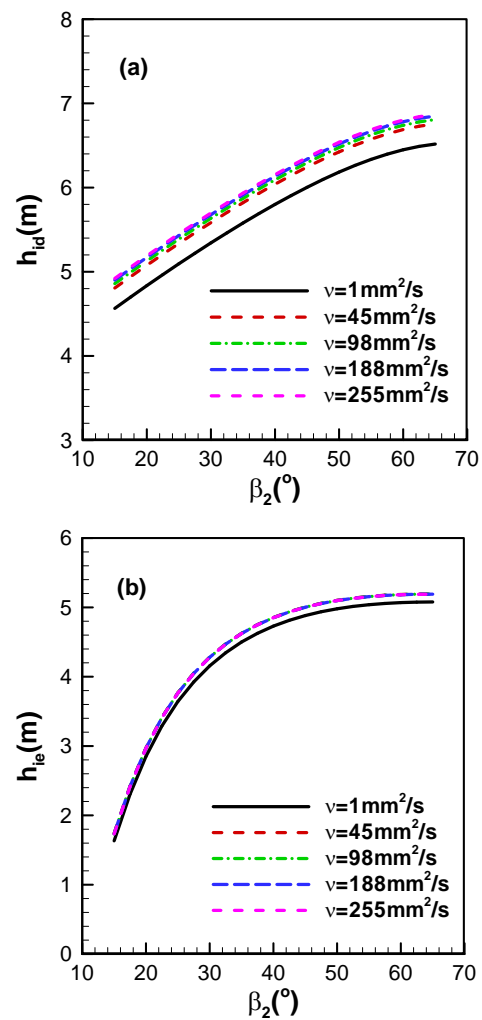

Fig. 14 Estimated diffusion (a) and sudden expansion as well as mixing losses (b) at BEP in terms of blade exit angle
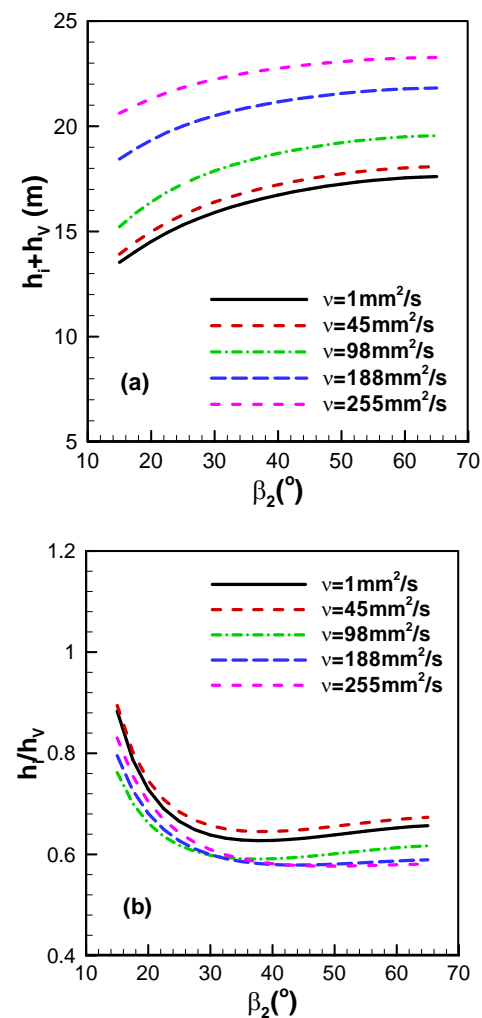

Fig. 15 Total head loss (a) and ratio of hydraulic losses in impeller to those in volute (b) at BEP in terms of blade discharge angle
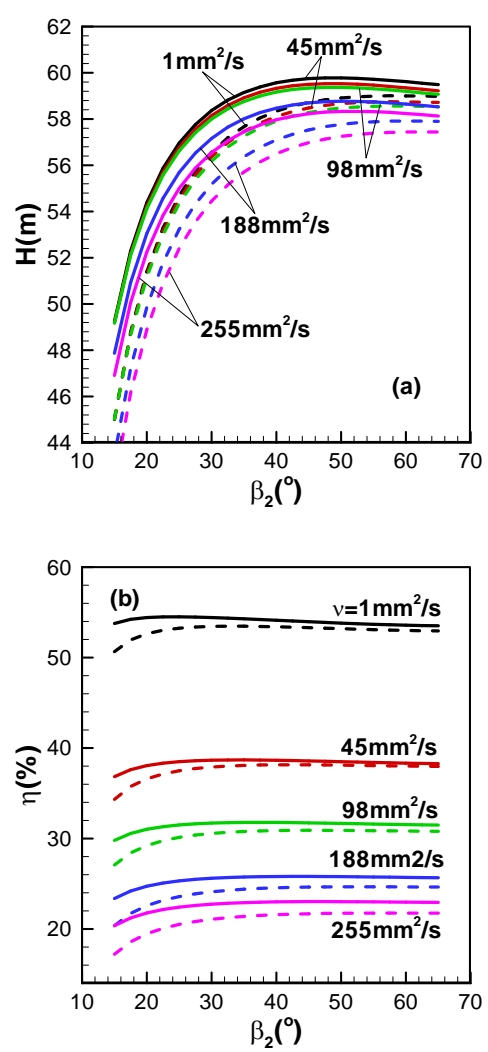

Fig. 16 Comparison of head (a) and efficiency (b) between original and redesigned cases, solid line is for redesigned case and dashed line is for original case

At the viscosity of $45 \mathrm{~mm}^{2} / \mathrm{s}$, the ratio of hydraulic loss is higher than that at $1 \mathrm{~mm}^{2} / \mathrm{s}$. The reason underlying this effect is that the diffusion loss in the impeller is increased remarked at $45 \mathrm{~mm}^{2} / \mathrm{s}$ shown in Fig. 14(a).

The experiments on the model pumps with two impellers of $23^{\circ}$ and $65^{\circ}$ discharge blade angles and 107 specific speed (Toyokura, Kurokawa and Kanemoto, 1979), the pump with the impeller of $65^{\circ}$ exit angle showed a better efficiency than that of $23^{\circ}$ angle at a viscosity of $45 \mathrm{~mm}^{2} / \mathrm{s}\left(R e_{2}=3.58 \times 10^{5}\right)$ yet. However, in our case, the pump with the impeller of $60^{\circ}$ discharge angle demonstrated a higher efficiency compared to that of $25^{\circ}$ angle at a viscosity as high as $255 \mathrm{~mm}^{2} / \mathrm{s} \quad\left(R e_{2}=1.37 \times 10^{4}\right)$. The effect of blade discharge angle on the pump performance in our case doesn't seem to be stronger at various viscosities. The reason for this may be the specific speed of the industrial pump used in the experiments is just 41.6, less than half of the specific speed of the model pumps. The specific speed effect needs to be investigated in future.

Since the friction loss in the volute is primary as shown in Fig. 12 (b), the volute cross-section area is enlarged by $44 \%$ (width and height are increased by $20 \%$, respectively) to reduce such a loss. In order to keep the ratio $b_{2} / b_{3}$ unchanged, the width of blade exit $b_{2}$ also is increased by $20 \%$. Fig. 16 illustrates the comparison of head and efficiency between the original and redesigned 
cases. It is clear that the pump with increased volute cross-section and enlarged blade exit width does get a better performance, especially at small blade exit angle $\left(\beta_{2} \leq 35^{\circ}\right)$. The hydraulic diameters of the volute and impellers are increased to $28.4 \mathrm{~mm}$ and $19.8 \mathrm{~mm}$ from $24.6 \mathrm{~mm}$ and $18.4 \mathrm{~mm}$, respectively. As a result, the peak pump efficiency is improved by $1 \%$ and the head is increased by $3.7 \%$ when $\beta_{2}=25^{\circ}$.

\section{CONClusion}

The effects of blade discharge angle on the performance of a centrifugal oil pump have been investigated experimentally and analytically while the pump handles water and other viscous oils. The hydraulic loss in the impeller and volute has been calculated in terms of both viscosity and blade exit angle by using a model on the fluid mechanics base. The following conclusions can be drawn: (1) the blade discharge angle has a strong but equal influence on the head, shaft power and efficiency of the centrifugal oil pump at various viscosities of liquids pumped; (2) The rapid reduction in the hydraulic and mechanical efficiencies is responsible for the pump performance degradation with increasing viscosity of liquids; (3) at all the viscosity values, the volute shows significant effect on the performance, it should be designed as perfectly as we can; (4) the theoretical hydraulic loss model is able to get broadly similar behaviour to the experimental curves; (5) for the hydraulic loss model is subject to limitations, it has been planned that the effect of blade exit angle will be investigated numerically by using CFD code, such as Fluent. It is hopeful the results will be available soon.

\section{ACKNOWLEDGEMENTS}

The research was conducted under a grant awarded through the Key Research and Development Program for the Quality Research Groups at Lanzhou University of Technology. The university was acknowledged indeed; additionally the previous postgraduate student FYL and technicians FZS and CX were also appreciated very much for their fantastic contributions in laboratory.

\section{REFERENCES}

Aisenstein, M.D. (1927). A new method of separating the hydraulic losses in a centrifugal pump. ASME Transactions 49, 1-7.

Aly, S. and S. Al-Zubaidy (1992). Performance prediction method for centrifugal pumps. SAE paper 921731.

Aoki, K., H. Ohta, and Y. Nakayama (1987). Study on centrifugal pump for high viscosity liquids (the 1 st report, effect of impeller output angle and number of blades on the pump performance of closed type impellers). Proceedings of the School of Engineering, Tokai University 27(2), 151-158.

Aoki, K., T. Yamamoto, H. Ohta and Y. Nakayama (1985). Study on centrifugal pump for high viscosity liquids. Trans JSME, Series B 51(468), 2753-2758.
Brennen, C.E. (1994). Hydrodynamics of Pumps. Oxford, UK, Oxford University Press, 48-51.

Fried, E. and I.E. Idelchik (1989). Flow Resistance: a Design Guide for Engineer. New York, USA: Hemisphere Publishing Corporation, 111-114.

Gulich, J.F. (1999a). Pumping highly viscous fluids with centrifugal pumps-part 1. World Pumps 395, 30-34.

Gulich. J.F. (1999b). Pumping highly viscous fluids with centrifugal pumps-part 2. World Pumps, 396, $39-42$.

Gulich. J.F. (2003). Effect of Reynolds number and surface roughness on the efficiency of centrifugal pumps. ASME Journal of Fluids Engineering 125, 670-679.

Hamkins, C. P. and P.H. Hergt (1987, May). Prediction of viscosity effects in centrifugal pumps by consideration of individual losses. In Proceedings of the Third European Congress on Fluid Machinery for the Oil, Petrochemical and Related Industries, Hague, Netherlands, pp. 207-217.

Iguchi. M. and M. Ohmi (1986). Loss coefficients foe flows through a sudden expansion and a sudden contraction closely placed. Trans JSME, Series $B$ 52(481), 3252-2258.

Kamimoto, G. and Y. Matsuoka (1956). On the flow in the impeller of centrifugal type hydraulic machinery (the 2nd report). Trans JSME, Series 3 22(113), 55-59.

Kurokawa, J. (1990, April). Simple formulae for volumetric efficiency and mechanical efficiency of hydraulic machinery. In Proceedings of the $3 r d$ Japan-China Joint Conference on Fluid Machinery, Osaka, Japan, pp. 101-108.

Li, W.G. (2000). The "sudden-rising head" effect in centrifugal oil pumps. World Pumps 409, 34-36.

Li, W.G. (2002). The Influence of number of blades on the performance of centrifugal oil pumps. World Pumps 427, 32-35.

Li, W.G. (2004). A method analyzing the performance of centrifugal oil pumps. ASME Journal of Fluids Engineering 126(3), 482-485.

Ohta, H (1999). Effect of Reynolds number of slip factor of centrifugal pump for high viscosity liquids. Trans JSME, Series B 65(639), 3697-3704.

Nemdili, A. and D.H. Hellmann (2007). Investigations on fluid friction of rotational disks with and without modified outlet sections in real centrifugal pump casing. Forsch Ingenieurwes 71, 59-67.

Oh, H.W. and M.K. Chung (1999). Optimum values of 
W.G. Li / JAFM, Vol. 4, No. 2, Issue 1, pp. 105-119, 2011.

design variables versus specific speed for centrifugal pumps. Proc Instn Mech Engrs, Part A 213, 219-226.

Ohta, H., and K. Aoki (1990). Study on centrifugal pump for high-viscosity liquids (effect of impeller output angle on the pump performance). Proceedings of the School of Engineering, Tokai University 30(1), 175-183.

Ohta, H. and K. Aoki (1996). Effect of impeller angle on performance and internal flow of centrifugal pump for high-viscosity liquids. Proceedings of the School of Engineering, Tokai University 36(1), 159-168.

Oliveira, P.J. and F.T. Pinho (1997). Pressure drop coefficient of laminar Newtonian flow in axisymmetric sudden expansions. International Journal of Heat and Fluid Flow 18(5), 518-529.

Oliveira. P.J., F.T. Pinho and A. Schulte (1998). A general correlation for the local loss coefficient in Newtonian axisymmetric sudden expansions. International Journal of Heat and Fluid Flow 19, 655-660.

Pigott, R.J. (1945). Prediction of centrifugal-pump performance, ASME Transactions, 67, 439-449.

Qiu, X.W., C. Mallikarachchi and M. Anderson (2007, May). A new slip factor model for axial and radial impellers. In Proceedings of GT2007-Power for Land, Sea and Air, Montreal, Canada.

Rathod, M.S. and F.M. Donovan (1980, March). Performance evaluation of a centrifugal cardiac pump. In Proceedings of the Conference on Performance Prediction of Centrifugal Pump and Compressors, New Orleans, USA, pp. 235-243.

Schlichting, H. (1968). Boundary-Layer Theory. New York, USA: McGraw-Hill Company, 574.

Stepanoff, A.J. (1948). Centrifugal and Axial Flow Pumps. New York, USA: John Wiley \& Sons, 193-199.

Stirling, T.E. (1982). Analysis of the design of two pumps using NEL methods, Centrifugal pumpsHydraulic design. London, UK: Mechanical Engineering Publication Ltd, 55-73.

Takagi, T.J., J. Kobayashi, H. Miyashiro, and H. Morimoto (1980, March). Performance prediction of single-suction centrifugal pumps of different specific speeds, In Proceedings of the Conference on Performance Prediction of Centrifugal Pump and Compressors, New Orleans, USA, pp. 227234.

Tanaka, K. and H. Ohashi (1984). Performance of centrifugal pumps at low Reynolds number (1st report, experimental study). Trans JSME, Series B
50(449), 279-285.

Toyokura, T., J. Kurokawa and T. Kanemoto (1979). Performance improvement for centrifugal pump handling high viscosity oil. Turbomachinery $7(2)$, 8-15.

Varley, F.A. (1961). Effects of impeller design and surface roughness on the performance of centrifugal pumps. Proc Instn Mech Engrs 175(21), 955-969.

White, F.M. (1994). Fluid Mechanics. New York, USA: McGraw-Hill INC, 339-340.

Yoon, E.S., H.W. Oh, M.K. Chung and J.S. Ha (1998). Performance prediction of mixed-flow pumps. Proc Instn Mech Engrs, Part A 212, 109-115.

Zaher, M.A. (2001). Approximate method for calculating the characteristics of a radial flow pump. Proc Instn Mech Engrs, Part E 215, 295316. 